public n'en font pas partie! Nous savons gré à l'auteur de nous en avoir épargnés. Ce sont: prophétie, prédiction, prévision, projet, plan, programme et finalement, l'objet du livre : prospective. Cette dernière comprend trois famille : la cognitive, la participative et la stratégique. Pour l'appliquer à un territoire, il importe d'en connaître la signification. Pour de Courson, le territoire correspond à un espace géographique concret quel qu'en soit sa taille, son échelle, son périmètre pour autant qu'on puisse y faire un travail prospectif. Mais l'auteur juge utile de le préciser, un territoire est fait d'une multitude d'acteurs aux intérêts souvent divergents et parfois opposés, hélas. Un territoire, tel un éléphant, ça bouge lentement, voire énormément comme le disait cette chanson de mon enfance. Les résistances au changement se posent inévitablement. Bien sûr, si le développement local ne se décrète pas, il en va ainsi pour les territoires que l'on ne peut changer par décret. Il faut en identifier les enjeux à l'aide d'une batterie de questions : quels sont les problèmes essentiels reliés à l'avenir du territoire étudié ? Quels sont les jeux de pouvoir à l'œuvre? Qui décide de quoi ? Quels sont les rapports de force ? Qui dispose des marges de manœuvre ? Ce type d'interrogation boucle la première étape de la démarche prospective.

Dans la deuxième partie de son ouvrage, de Courson offre les clés d'un apprentissage concret de la prospective et, ce faisant, il cherche à répondre aux sempiternelles questions : "Comment ça marche? (Au Québec nous dirions : qu'est-ce que ça mange en hiver ?) À quoi ça sert ? Et moi dans tout ça ? " Le tout débute par un exemple on ne peut plus francofrançais. On connaît l'importance des vacances pour les Français. Pour eux, le mot se conjugue nécessairement avec le verbe « partir ». Si vous dites à un Français que, durant vos vacances, vous allez jouer au golf, au tennis, faire du vélo, aller à la piscine, promener vos petits-enfants, rencontrer des amis, profiter des festivals qui se suivent, etc. sans quitter votre lieu de résidence, vous vous ferez répondre inévitablement: " Mince alors!!! Ce n'est pas des vacances çaaaaaaaaa! ». Alors, de Courson évoque le cas des Dupuis qui, eux, partent en vacances, tous les ans, le 15 juillet, en traînant leur tente dans une petite remorque, pour se rendre, tel Monsieur Hulot (merci Tati), au même camping, et si possible au même emplacement, pour y retrouver les mêmes gens qu'ils se permettent de tutoyer. Mais voilà que, par un heureux hasard, M. Dupuis obtient une promotion accompa- gnée d'une généreuse augmentation de salaire. Tout change alors : et le statut social et le moyen de transport (camping car). Faut donc faire, pour l'été suivant, une véritable opération de prospective afin de se conformer à la nouvelle donne. Et il en ira ainsi pour l'achat d'une maison. En somme, la prospective fait partie de la vie courante de ceux qui ont les moyens de la mettre en pratique. L'auteur le montre avec humour et habileté.

Comme il l'écrit, dans ces " histoires du futur», le mouvement de la pensée est toujours le même : partir de la situation présente dans sa dynamique; examiner ce qui est en tension, potentialités, menaces, dangers et tendances lourdes; basculer vers le futur en ouvrant des pistes, provoquant des alternatives, imaginant des scénarios. Et tout ceci peut se faire en marchant (moi je préfère en pédalant). Ainsi, c'est en marchant que notre auteur, tel qu'il le présente, procède au diagnostic d'une ville. Son œil observateur le conduit à s'interroger sur les évolutions structurelles lourdes; sur les risques, les tensions, les ruptures possibles; sur les opportunités, les avancées positives, les lignes d'espoir. Terminons par ce clin d'œil au pays de Pele : le Brésil a-t-il un avenir?, s'interroge de Courson. Comment ne pas répondre positivement sur la base de trois atouts stratégiques : sa masse, son territoire et sa culture. Eh oui, et c'est faire abstraction du football et du volley de plage...

En conclusion, on peut lire que la prospective n'est ni une science, ni un art, c'est une recherche des futurs possibles. L'auteur a réussi son pari : donner à son lecteur l'envie de participer au processus susceptible de façonner son futur. Et j'ajouterais que la lecture de son volume ne rend que plus agréable celui qui suit.

\section{Bernard Guesnier \& Christian Lemaignan Connaissance, solidarité, création : le cercle d'or des territoires Paris, L'Harmattan, collection Administration et aménagement du territoire, 2006, 299 pages}

Le lien avec l'ouvrage précédent se fait aisément quand on lit dès le tout début qu'une fois que le territoire aura fait son deuil de sa crise (de la quarantaine ?), il s'agit d'en chercher les conditions de cercles vertueux : transformer les handicaps en atouts et saisir 
des opportunités, tout en en écartant les menaces. En d'autres termes, écrivent mes collègues Guesnier et Lemaignan de l'Université de Poitiers, il importe d'identifier dans le fonctionnement et l'évolution d'un système socio-économique localisé ce qui est imputable aux tendances lourdes et ce qui relève de la gouvernance territoriale mobilisant partenariat et solidarité. Ce livre découle d'une étude dont le double objet était :

- de fournir un «kit d'informations » aux responsables locaux pour renforcer leur capacité d'expertise et de diagnostic socio-économique;

- d'analyser le jeu des acteurs pour mieux appréhender la dynamique des territoires.

Disons-le sans tarder, nos amis poitevins ont su parfaitement atteindre les objectifs qu'ils se sont fixés.

Toujours en introduction, les auteurs nous font prendre conscience que, ô combien, le temps passe vite. Ainsi, à la suite d'une allusion à la crise du milieu des années 1970, causée (ou accompagnée) par les deux chocs pétroliers, on présente les années 1980 comme étant caractérisées par la préoccupation des territoires d'accumuler des connaissances. On sait qu'une abondante littérature de cette époque se rapporte aux technopoles, aux districts industriels (italiens surtout), aux pépinières d'entreprises et autres formes d'incubation. Une décennie plus tard, comme signalé, les territoires attacheront une importance particulière à l'organisation, à l'animation des acteurs, aux effets réseaux, en somme à tout ce qui dynamise un milieu. On trouve ici un beau clin d'oeil à notre ami Pecqueur : "Les entreprises ne sont pas sur un territoire, elles naissent du territoire ». Enfin, avec l'avènement du nouveau siècle, de nombreux territoires, comme ce fut le cas trente ans plus tôt, se trouvent en ruptures d'activités, soulignent les auteurs. Pour eux, c'est le temps de la Créativité dans tous les domaines à la faveur d'une certaine démocratie participative. Et, comme le fait remarquer notre Jacques Proulx national (Solidarité rurale), ces innovations incluent les saveurs des terroirs (comme la bière de Sarasin de Saint-Paulin en Mauricie). Cette nouvelle approche, aux yeux des auteurs, sous-tend une pensée complexe, étayée de responsabilité sociale qui donne tout son sens à l'expression trop souvent galvaudée de «développement durable $»$.
Le concept d'alliance, impliquant un climat de confiance et la pérennité, justifie la référence à un cercle d'or que l'on souhaite, il va sans dire, le plus vertueux possible. Il comprend trois composantes : la Créativité (capital culturel), la Solidarité (capital social) et la Connaissance (capital humain). Leurs interrelations s'effectuent à l'échelon du « local» vu comme un lieu politique où le développement prend forme grâce à des acteurs locaux agissant en tant que catalyseurs des potentialités existantes. Évoquer les acteurs locaux, on le pense bien, conduit au concept de gouvernance défini ici comme un système démocratiquement organisé par lequel les citoyens agissent collectivement à l'échelon local en vue de l'amélioration de leur bienêtre, tout en promouvant la justice sociale.

Le premier chapitre, en portant sur les « territoires cognitifs », intéressera plus particulièrement les lecteurs d'O\&T. On retrouve ici la belle conception de Jambes pour qui le territoire se veut un principe organisateur approprié. Ce territoire, il évolue en « apprenant ». Ce qui fait penser à l'image du tuteur attaché à l'arbre pour en favoriser la meilleure croissance possible. Il revient aux acteurs locaux de faire en sorte que leur territoire assimile au mieux les connaissances mises à sa disposition. Pour Bernard Guesnier et Christian Lemaigan, concevoir le territoire comme un système socio-économique complexe exige le recours à des moyens méthodologiques permettant de le connaître pour mieux comprendre son fonctionnement afin d'anticiper son évolution. Reste la sempiternelle question de l'échelon d'intervention : quel est le périmètre pertinent? La France a ses découpages administratifs et le Québec a les siens. Dans les années 1970, nous avions nos commissariats industriels et leur aire d'intervention dont l'équivalent français (après des recherches personnelles) se trouvait sous la forme de conseils d'expansion économique. Les premiers sont devenus des CLD (Centres locaux de développement) intervenant au niveau des MRC (Municipalités régionales de comté). Hélas, les auteurs ne disent pas ce que sont devenus les seconds. Le chapitre se termine par une belle définition de l'intelligence territoriale : l'activité de traitement de l'information en vue d'une décision territoriale.

Le deuxième chapitre débute avec une évocation à un nouveau modèle de développement étant donné l'importance que la solidarité sociale y occuperait. Suis-je vieux jeu ? C'est toujours avec scepticisme que je lis ces allusions à de soi-disant nouvelles réalités : nou- 
velle géographie économique, nouvelle économie sociale... nouvelle cuisine (dans ce dernier cas, c'est, hélas, une réalité). Reprenant leur cercle d'or, les auteurs y rattachent cette fois pas moins de douze composantes pour décrire ce qu'est un territoire solidaire. On tourne une roue qui va de la richesse à la gouvernance, en passant, entre autres, par les projets et la cohésion sociale. Des travaux en cours sur les Pactes ruraux, élément-clé de la politique québécoise de la ruralité, permettront peut-être d'identifier de tels territoires dits solidaires.

Certains de mes collègues de 1'Université du Québec à Montréal et de l'Université du Québec en Outaouais, très portés sur l'économie sociale, apprécieront les nombreuses allusions faites à cette (nouvelle ?) discipline à laquelle les auteurs accolent l'économie solidaire vue comme l'ensemble des initiatives qui se développent autour de valeurs de réciprocité, de citoyenneté et de collectivité. Ce qui rappellera aux gens de ma génération ce que l'on désignait à une certaine époque sous l'appellation « économie communautaire ».

La section «Comprendre la dynamique des territoires » à l'intérieur du troisième et dernier chapitre portant sur les territoires créatifs contient un grande nombre d'illustrations présentées sous la forme de radioscopies. Ainsi, à titre d'exemple, à Dieppe, en relation avec la logique des réseaux, on signale pour le port de pêche 1747 tonnes de coquilles Saint-Jacques, 2700 tonnes de poisson, 53 bateaux, 256 marins (sic) et un nombre indéterminé de mareyeurs... On comprendra que le lecteur est ici invité à tourner les pages à la recherche de l'essentiel. Et il trouvera, entre autres, dans une section sur la nécessité de penser les territoires à travers les logiques d'acteurs, un intéressant quadrant mettant en relation territoire projet - territoire créatif - territoire soumis - territoire réceptacle. Le tout se termine de façon brusque, sans la moindre conclusion, par un schéma voulant montrer que l'économie cognitive favorise l'échange des savoir, alors que l'économie sociale et solidaire rappelle que la société du vivre ensemble met l'homme au centre du sujet. Au moment de la «montée en puissance » de l'économie libérale (milieu du $\mathrm{XIX}^{\mathrm{e}}$ siècle), Thomas Carlyle disait que la théorie qui lui servait de base n'était rien d'autre qu'une science funeste (dismal science). À la lecture de ce livre, il admettrait que la science économique peut être humaine.

\section{Christian Schmitt (sous la direction de) \\ Université et entrepreneuriat : une relation en quête de sens Paris, L'Harmattan 2005, 315 pages}

Voici un volume qui traite de l'université comme une organisation susceptible d'intervenir pour le mieux être de son territoire via l'entrepreneuriat. De « quoi » s'agit-il ? Et « comment» parvenir à établir une relation entre l'université et la création d'entreprise ? Tel est le questionnement sous-jacent de cet ouvrage auquel pas moins de dix collègues ont été appelés à collaborer. Le responsable de l'ouvrage, Christian Schmitt, docteur en gestion, est maitre de conférences à l'ENSAIA (École nationale supérieure d'agronomie et des industries alimentaires) à l'INPL (Institut national polytechnique de Lorraine) et chercheur au GREFIGE (Groupe de recherche en économie financière et gestion des entreprises) (la France et ses sigles...) à la ville des bergamotes (bonbons qui font la renommée de Nancy). C'est sans surprise que l'on trouve dans sa note biographique une allusion à ses travaux sur la relation entre l'université et l'entrepreneuriat. Il est aussi mentionné qu'il fut professeur invité à l'INRPME (Institut de recherche sur les PME) de l'UQTR (Université du Québec à Trois-Rivières), avec les conséquences que je mentionne plus loin. Cet ouvrage, tel que bien précisé en introduction, se veut être une vitrine des pratiques dans les domaines de la formation, de la recherche et de la valorisation de l'entrepreneuriat au sein de l'université. Pour ce faire, on offre au lecteur des expériences menées en Iran, au Vietnam, au Maroc, en Turquie, au Mexique, en Belgique et finalement... au Québec. Pas en France ? Enfin, nous pouvons toujours croire que la mère-patrie fait partie des réflexions, de façon implicite, que nous fournit notre collègue lorrain dans un intéressant chapitre qu'il co-signe avec deux collaborateurs.

Il serait fastidieux de résumer la gamme variée des initiatives mises en évidence ici et là à la faveur des différents chapitres. Elles intéressent avant tout les acteurs directement impliqués dans une telle démarche pour voir les similitudes et surtout, tel qu'indiqué, toujours en introduction, les distinctions. Le lecteur intéressé trouvera à n'en pas douter des leçons à tirer de nature à voir une mise en application, toute chose étant égale par ailleurs, comme le veut l'adage si cher aux économistes (citeris parebus). Robert Paturel, 